\title{
FAILURE CRITERIA OF DENTIN-RESIN ADHESION-A MIXED MODE FRACTURE MECHANICS APPROACH
}

\author{
Chun-Pin Lin ${ }^{1}$, Jung-Ho Cheng ${ }^{2}$, Antheunis Versluis ${ }^{3}$ and William H. Douglas ${ }^{3}$ \\ ${ }^{1}$ School of Dentistry, College of Medicine, National Taiwan University, Taipei, Taiwan, Republic \\ of China ${ }^{2}$ Department of Mechanical Engineering, College of Engineering, National Taiwan \\ University, Taipei, Taiwan, Republic of China ${ }^{3}$ Minnesota Dental Research Center for Biomaterials \\ and Biomechanics, School of Dentistry, University of Minnesota, Minneapolis, MN
}

(Received September 9, 1999)

(Accepted in revised form October 20, 1999)

Keywords: Biomaterials; Composites; Fracture toughness

\section{$\underline{\text { Introduction }}$}

Intact and restored teeth are laminated structures where tissues and materials of widely different properties are bonded together, thus preserving the integrity of the entire dental structure. These interfaces are under challenge in daily functions of the oral cavity, which include the comminution of food under significant interdental forces. There is a growing awareness that adhesion is of first importance in restorative dentistry. By the same token there is an urgent need for a standardized test for interfacial strength, which can predict clinical dental performance of new materials and reveal structure-property relationships that succeed (1). Evaluations of dental adhesive performance are most often based on measurements of bond strength determined in shear or tensile loading. Adhesive strength is simply defined as the load at failure divided by the cross-sectional area of the bonded surface. Previous studies have demonstrated that a non-uniform stress distribution at the interface is produced during tensile or shear bond testing, and the so called "average stress" or "nominal stress" of conventional tensile or shear bond strength is therefore not representative of the true failure stress generated at the interface $(2,3)$. It has been suggested that a more promising approach to determine the bond strength be based on interfacial fracture mechanics, which represents the real local effort of the creation in a new crack surface $(4,5)$.

In dental research most of the fracture mechanics efforts have been occupied with the propagation of cracks under opening mode conditions (Mode I). However, there is an increased awareness that numerous fracture problems are inherently mixed mode (6). In interfacial fracture mechanics this can be caused by the elastic mismatch across an interface, while microstructural properties could also lead to the mixed mode conditions at the crack tip.

So far, there is no generally accepted fracture mechanics based interfacial fracture toughness test. The purpose of this study is to define the biomechanics of the human dentin-resin interface by a mixed mode fracture mechanics approach. A finite element model for this newly proposed mixed mode dentin-resin adhesion fracture test was created and verified. This model is intended to provide a theoretical basis for an experimental fracture test that will shed light on the mechanism of dentin-resin adhesion.

\footnotetext{
*Corresponding author.
} 


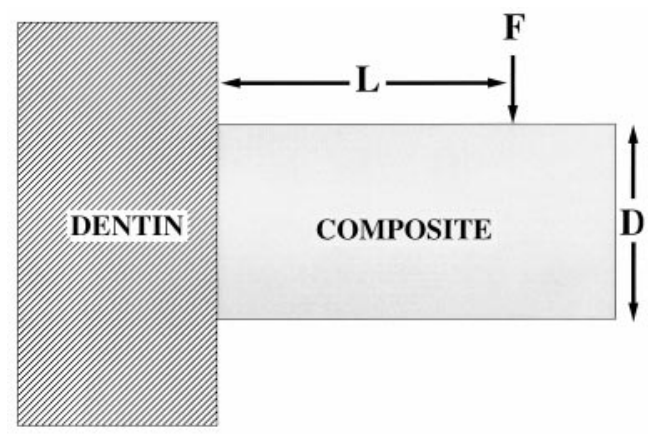

Figure 1. Diagrammatic representation of the mixed mode adhesion fracture test specimen. F, applied load; D, diameter of composite rod; L, loading length.

\section{Geometry of the Proposed Mixed Mode Fracture Specimen}

The proposed mixed mode fracture test specimen is related to current used stress-based shear tests, but is based on a fracture mechanics approach. The test specimen configuration with basic notations is shown in Figures 1 and 2. The specimen contains a chevron $\mathrm{V}$ with subtended angle $2 \theta$. The apex position is given by $a_{0}$ and the chevron ends at $a_{1}$. With a crack present, the instantaneous crack front length $(b)$ is

$$
b=2\left(a_{1} D-a_{1}^{2}\right)^{0.5} \cdot \frac{a-a_{0}}{a_{1}-a_{01}}=2\left(\alpha-\alpha_{0}\right) \tan \theta
$$

when $a_{0} \leq a \leq a_{1}$, i.e. inside the chevron, where $D$ is the diameter of the composite rod. With $2 \theta=$ $90^{\circ}$, we obtain $b=2\left(a-\mathrm{a}_{0}\right)=2 D\left(\alpha-\alpha_{0}\right)$. In this study, $D=4 \mathrm{~mm}$ and $b=2\left(a_{1}-\mathrm{a}_{0}\right)=2 \mathrm{D}$ $\left(\alpha_{1}-\alpha_{0}\right)=3.8576 \mathrm{~mm}$, and $\alpha=a / D, \alpha_{1}=a_{1} / D=0.632$, and $\alpha_{0}=a_{0} / D=0.15$.

\section{Interfacial Chevron Notch Fracture Mechanics}

As depicted in Figure 2, the chevron notch has a V-shaped ligament such that the notch width varies through the length, $a$. When the crack length $a=a_{0}$, the stress intensity factor at the vertex of the chevron is very high, since a finite load is applied over a very small net thickness. The crack is stable

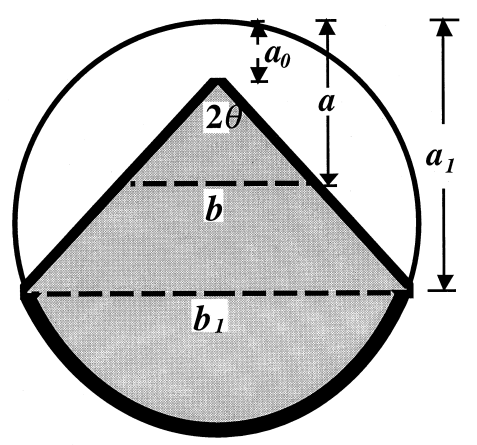

Figure 2. Geometric illustration of the chevron notch bonded area. $a$, crack length; $a_{0}$, initial crack length; $a_{1}$, maximum depth of chevron flanks; $2 \theta$, subtended the chevron angle; $b$, instantaneous crack front width; $b_{1}$, crack front width at the chevron end. 
at this point, because the driving force decreases at the crack advances; thus, additional load is required to grow the crack further. The critical load in the test, $F_{\mathrm{c}}$, is achieved when the crack growth reaches $\mathrm{a}_{\mathrm{c}}$ (critical crack length). The critical crack length corresponds to the minimum stress intensity factor coefficient or geometric factor coefficient. At this point, the specimen will become unstable, which leads to catastrophic fracture. In real life, both stable crack and catastrophic fracture may happen at resin-dentin interfaces. Consequently, the mixed mode, chevron-notched, interfacial fracture test may simulate fracture surfaces that occur in the natural fracture situations.

\section{$\underline{\text { Interfacial Fracture Energy Release Rate }}$}

The expression for mixed mode adhesive fracture toughness, $G_{\mathrm{C}}$, is based on the strain energy theory. The energy required advancing a crack an increment, $d a$, is given as

$$
d W=G_{C} \cdot b \cdot d a
$$

where $G_{\mathrm{C}}$ is the mixed mode critical strain energy release rate and $b$ is the instantaneous crack front width at a critical crack length, $a+d a$. Irrecoverable work, $d W$, when the crack advances an amount $d a$, can be expressed in terms of the elastic compliance change while loading the material. Thus the irrecoverable work is expressed as

$$
d W=\frac{1}{2} \cdot F_{C} \cdot d Z=\frac{1}{2} \cdot F_{C}^{2} \cdot d C
$$

where $F_{\mathrm{C}}$ is the critical failure load, $d C$ is the incremental change in compliance and $d Z$ is the incremental change in loading displacement due to crack advance, $d a$. Combining equations (1) and (2) will give the critical strain energy release rate as the classical compliance equation from linear elastic fracture mechanics (LEFM) (7). That is,

$$
G_{C}=\frac{F_{C}^{2} \cdot d C}{2 b \cdot d a}
$$

Note that in deriving Eqs. $(2) \sim(4)$, there are no assumptions requiring only one material in this system. In a bimaterial system, because of the elastic mismatch of the bonded substrates, the interface fracture problems are inherently mixed mode (combination of tension and shear). Accordingly, a bimaterial interfacial fracture energy, $G_{\mathrm{C}}$, includes not only the tensile opening fracture mode (Mode I) but also the in-plane shear fracture mode.

The total incremental change in displacement of loadline is a combination of the displacement caused by composite bending and dentin compression. Thus, the Eq. (4) becomes

$$
G_{C}=\frac{F_{C}^{2}}{2 b} \cdot \frac{d C_{1}+d C_{2}}{d a}
$$

The subscripts 1 and 2 refer to composite and dentin, respectively. The same delimitation will be used throughout this paper.

The notched section of the mixed mode adhesive fracture specimen is a chevron $\mathrm{V}$ with a subtended angle $2 \theta=90^{\circ}$. Combining Eqs. (1) and (5), one obtains the following relationship:

$$
G_{C}=\frac{F_{C}^{2}}{4\left(a-a_{0}\right)} \cdot \frac{d C_{1}+d C_{2}}{d a}
$$


which can be rearranged into

$$
G_{C}=\frac{F_{C}^{2} L^{2}}{D^{5}}\left(\frac{Y_{1 m}}{E_{1}}+\frac{Y_{2 m}}{E_{2}}\right)
$$

where $E_{1}$ and $E_{2}$ refer to the elastic moduli of composite and dentin, respectively, and $Y_{1 \mathrm{~m}}$ and $Y_{2 \mathrm{~m}}$ are the geometric factor coefficients.

$$
Y_{1}=\frac{d C_{1}\left(D^{3} / L^{2}\right) E_{1}}{4 d \alpha\left(\alpha-\alpha_{0}\right)} ; \quad Y_{2}=\frac{d C_{2}\left(D^{3} / L^{2}\right) E_{2}}{4 d \alpha\left(\alpha-\alpha_{0}\right)}
$$

The main purpose for writing strain energy release rate as Eq. (7) is that according to LEFM (8), the variation of compliance is proportional to $\left(L^{2} / D^{3}\right)$ of the specimen. Therefore, the quantities of $Y_{1}$ and $Y_{2}$ are dimensionless functions of the ratio $a / D(=\alpha)$. They attain the minimum when the crack growth reaches the critical crack length and becomes unstable thereafter. They are independent of the specimen material as long as the scaled specimen configuration remains constant.

\section{Phase Angle ( $\Psi)$}

One important feature of bimaterial interface cracks is that the phase angle, $\Psi$, is often non-zero; even when the external loading is normal to the interface plane (9). This situation arises because of the elastic mismatch across the interface. Consequently, when solving interface fracture problems, it is essential to calculate $\Psi$. This feature of interface fracture represents the major difference from the familiar treatment of fracture within an elastically homogeneous medium. One approach for evaluating the phase angle $(\Psi)$ is the crack surface displacement (CSD) method (10). The crack surface displacements exhibit an oscillatory solution, i.e. for plane strain

$$
d \delta_{y}+i d \delta_{x}=\frac{2\left[\left(1-\nu_{1}\right) / u_{1}+\left(1-\nu_{2}\right) / u_{2}\right]}{(1+i 2 \epsilon) \cosh (\pi \epsilon)}\left(K_{I}^{*}+i K_{I I}^{*}\right) \sqrt{\frac{r}{2 \pi}} r^{i \epsilon}
$$

where $\epsilon=\frac{1}{2 \pi} \log \left[\left(\frac{k_{1}}{u_{1}}+\frac{1}{u_{2}}\right) /\left(\frac{k_{2}}{u_{2}}+\frac{1}{u_{1}}\right)\right]$ and $i=\sqrt{-1}$. The parameters for the materials are such that $u_{j}$ are the shear moduli. The parameters $k_{j}$ are defined so that they equal $\left(3-\nu_{j}\right) /\left(1+\nu_{j}\right)$ in plane stress and otherwise are $3-4 \nu_{j}$. $\nu_{j}$ are the Poisson's ratios. It is important to note that the factors $K_{I}^{*}$ and $K_{I I}^{*}$ do not represent opening $\left(K_{I}\right)$ and shear $\left(K_{I I}\right)$ modes (11). Instead, the oscillatory nature and the associated displacements mean that the ratio of modes in the crack surface motions varies with distance from the tip. When the two materials are identical $(\epsilon=0)$ and the factors $K_{I}^{*}$ and $K_{I I}^{*}$ become identical to $K_{I}$ and $K_{I I}$, respectively. $\gamma$ is the distance from the crack tip. $d \delta_{\mathrm{x}}$ and $d \delta_{\mathrm{y}}$ now denote the relative displacements of two initially neighboring points on the crack surfaces behind the tip. We have chosen to introduce the elastic fracture mechanics for bimaterial systems with $\epsilon=0$, either exactly or as an approximation. With this assumption of $\epsilon=0$, the Eq. (9) becomes

$$
\left(\delta_{y}, \delta_{x}\right)=\left(K_{I}, K_{I I}\right) \sqrt{2 \pi r}
$$

The phase angle, which is a measure of the combination of shear and opening mode experienced by the interface crack surface, can be expressed by the following relation:

$$
\Psi=\tan ^{-1}\left(K_{I I} / K_{I}\right)=\tan ^{-1}\left(d \delta_{x} / d \delta_{y}\right)
$$




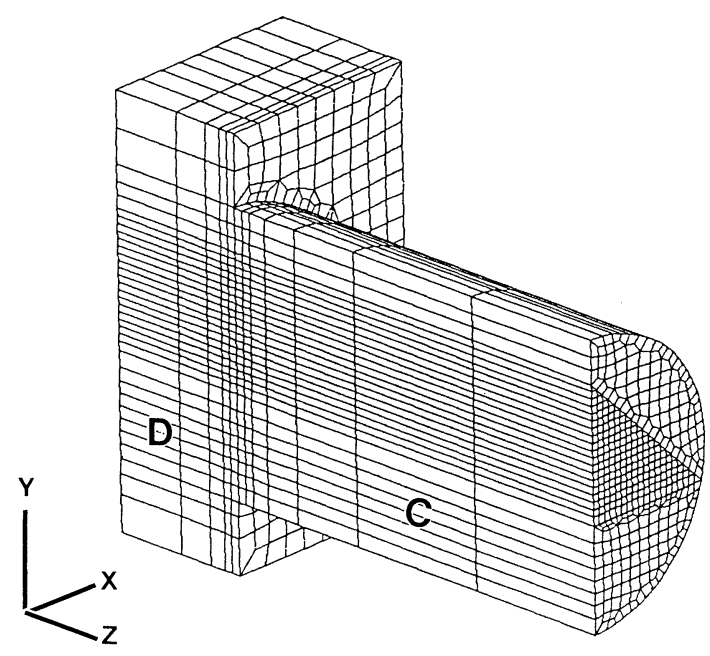

Figure 3. A three-dimensional finite element mesh for mixed mode dentin-resin interfacial fracture simulation, using isoparametric 8-noded hexahedron elements. Owing to symmetry, only half of the test specimen was modeled. C, composite resin; D, dentin.

\section{Theoretical Finite Element Analysis}

Because of the specimen symmetry, only half of the specimen was modeled, as shown in Figure 3. Isoparametric eight-noded hexahedron elements were used. In order to confirm the quality of the constructed mesh, a convergence study was conducted. For the convergence test, four meshes with various levels of refinements at the chevron-notched interface were made. All meshes were loaded at nodes on the composite rod with 4 different loadline lengths $(\mathrm{L}=1,2,4$, and $6 \mathrm{~mm}$ ). To simulate experimental conditions, the loadline node was restrained in $\mathrm{x}$ and $\mathrm{z}$ directions, whereas the nodes on the base and back of the dentin block $(2 \times 3 \times 6 \mathrm{~mm})$ were restrained in $\mathrm{x}$ and $\mathrm{y}$ directions. For both material aspects separate nodes were made at the chevron-shaped interface, for which the displacements were initially tied together across the interface. An advancing crack was simulated by releasing these tied nodes, starting from the vertex of the chevron notch, with $6 \sim 500 \mu \mathrm{m}$ increments (depending on the local mesh refinement) up to the end of the specimen. As a consequence, $4 \sim 20$ increments of loadline compliance as a function of crack length were computed. All models were solved using the MARC K6.1 finite element program (Nippon Marc Analysis Research Corporation, Tokyo, Japan) on a Silicon Graphics workstation (Indy, Silicon Graphics, Inc., Mountain View, CA) and a Cray J916 at the NCHC (National Center for High-Performance Computing, Taiwan, ROC). The output was post-processed using Mentat II v2.1.3 (Nippon Marc Analysis Research Corporation, Tokyo, Japan) on a graphics workstation (Silicon Graphics, Inc., Mountain View, CA). Convergence of the results was determined by plotting the minimum geometric factor coefficient, $Y_{m}$, with respect to the number of degree-offreedom (dof). $Y$ is calculated by Eq. (8).

The crack surface displacement method was utilized to determine the phase angles along the crack path with respect to 4 different loadline lengths. The finite element node was chosen through an internal consistency check which balanced the numerical error on the displacement in the near tip region. The phase angle is a measurement of relative displacements of two initially neighboring points on the crack surfaces behind the tip. 


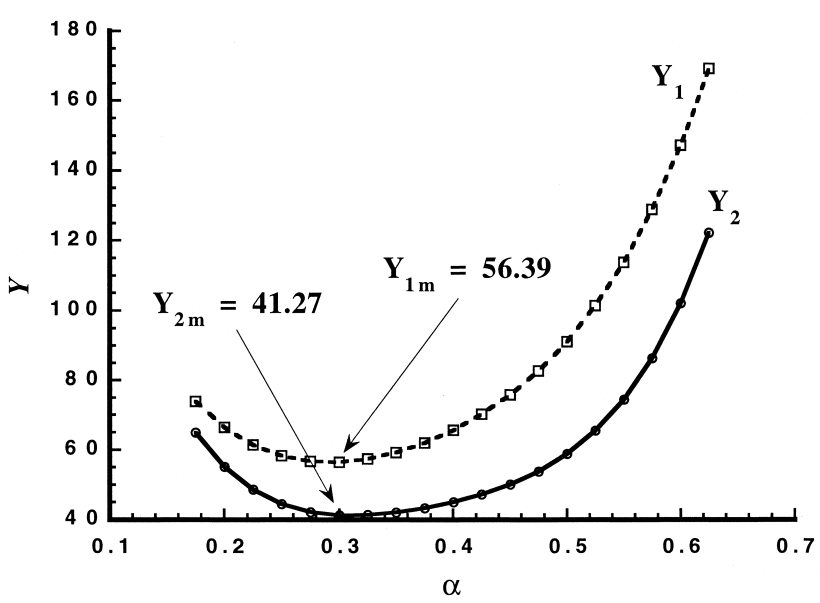

Figure 4. The geometric factor coefficients $(Y)$ with respect to the dimensionless loadline lengths $(\alpha)$. Subscripts 1 and 2 refer to composite and dentin, respectively. Subscript $\mathrm{m}$ indicates the minimum value.

\section{Results and Discussion}

The results of the convergence test showed that more accurate results were calculated for the geometric factor coefficients with the most refined mesh, Mesh 4 (Figure 3), which had 5605 elements, 6822 nodes and 20466 degrees of freedom. However, the differences in the calculated minimum geometric factor coefficients $\left(Y_{1 m}\right.$ and $\left.Y_{2 m}\right)$ were less than $1.87 \%$ between Mesh 3 and Mesh 4, whereas the dof increased by 3 times. We thus had confidence that the most refined Mesh 4 provided sufficient accuracy while maintaining sufficient detail and structure response. The logarithms of the basic compliance data could be fitted with a fourth-degree polynomial in dimensionless crack length $(a / D)$. The fitted curves were differentiated and the values of $Y_{1}$ and $Y_{2}$ were calculated from Eq. (8). The results are plotted in Figure 4. The minimum geometric factor coefficients are 56.39 for $Y_{1 m}$ and 41.27 for $Y_{2 m}$. These values are vital for calculation of the interfacial fracture energy of dentin-resin bonding. In addition, the dimensionless critical crack lengths were found to be approximately 0.3 for both $Y_{1 m}$ and $Y_{2 m}$. This represents a distance of $0.6 \mathrm{~mm}$ away from the vertex of the chevron notch.

The phase angles $(\Psi)$ were calculated using the finite element analysis of CSD method. Matos et al. (10) reported that the results from this crack surface displacement method using the finite element method were in excellent agreement with those obtained via the integral equation methods (12). The values obtained for these phase angles with respect to loadline lengths are depicted in Figure 5. The phase angle increased substantially with decrease in loadline length, especially when the loadline length was less than $2 \mathrm{~mm}$. This indicates that shear fracture mode (Mode II) becomes dominated as loadline moves towards dentin-resin interface. Conversely, the shear fracture mode was less significant when the loadline length was greater than $4 \mathrm{~mm}$, at which the phase angle was only 4 degrees or less. In this case, the fracture mode was dominated by tensile opening failure mode. It is believed that a unique relationship may exist between fracture energy and phase angle for a given interface. Therefore, the interfacial fracture energy is better characterized both in terms of a strain energy release rate and a phase angle of loading. For example, using a clinical available composite resin (elastic modulus $E=20 \mathrm{GPa}$ ) bonded to human dentin $(E=12 \mathrm{GPa})$ as an example, a critical fracture load of $23.63 \mathrm{~N}$ has been measured at $4 \mathrm{~mm}$ of loadline length. The interfacial fracture energy, calculated by Eq. (7), was: $G_{\mathrm{C}}=$ $54.62 \mathrm{Jm}^{-2}$ at the phase angle of $4.724^{\circ}$. 


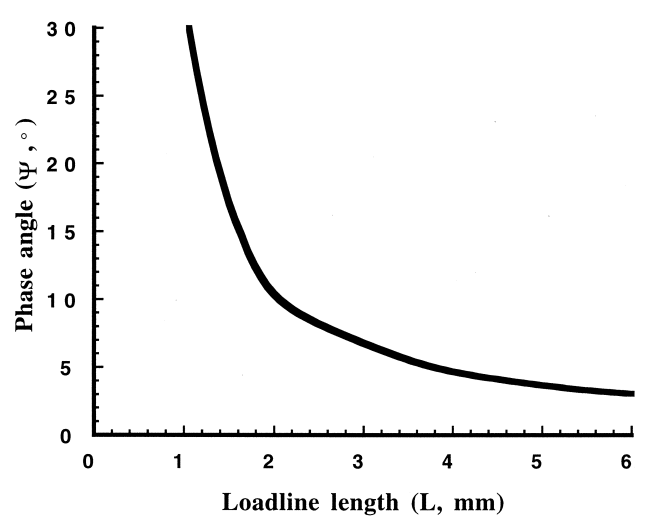

Figure 5. The effect of loadline length $(\mathrm{L})$ on the phase angle $(\Psi)$ at the critical crack line of dentin-resin bonded specimen.

\section{Conclusions}

A new test specimen has been proposed for application of mixed mode fracture mechanics to dentin-resin interfacial problems, supported by a rigorous theoretical finite element analysis. The results of this study show that this proposed test method is capable of measuring the mixed mode fracture resistance over an essential range of mode combination. The fracture energy of a bimaterial interface may vary appreciably for different loadline lengths. The phase angle of loading is strongly influenced by the choice of loadline length, especially when the load line length is less than $2 \mathrm{~mm}$. Consequently, use of loadline length greater than $2 \mathrm{~mm}$ is recommended. Since a unique relationship may exist between fracture energy and phase angle for a given interface, the interfacial fracture toughness is better characterized both in terms of a strain energy release rate and a phase angle of loading. However, an experimental fracture test of dentin-resin bonding based on this proposed method need to be performed to shed light on the contribution of phase angle on the fracture energy as well as on the adhesion mechanism of dentin-resin bonding.

\section{$\underline{\text { References }}$}

1. C. P. Lin and W. H. Douglas, J. Dent. Res. 73, 1072 (1994).

2. R. van Noort, et al., J. Dent. 17, 61 (1989).

3. A. Versluis, D. Tantbirojn, and W. H. Douglas, J. Dent. Res. 76, 1298 (1997).

4. C. P. Lin and W. H. Douglas, J. Biomech. 27, 1037 (1994).

5. C. P. Lin et al., Scripta Mater. 38, 115 (1998).

6. A. G. Evans and J. W. Hutchinson, Acta Metall. Mater. 37, 909 (1989).

7. L. M. Barker, Eng. Fract. Mech. 9, 361 (1977).

8. T. L. Anderson, Fracture Mechanics: Fundamentals and Applications, p. 50, CRC Press, Boca Raton, FL (1991).

9. J. R. Rice, J Appl. Mech. 55, 98 (1988).

10. P. P. L. Matos, et al., Int. J. Fract. 40, 235 (1989).

11. J. R. Rice, G. C. Sih, J. Appl. Mech. 32, 418 (1965).

12. Z. Suo, J. W. Hutchinson, Mater. Sci. Eng. A107, 135 (1989). 